\title{
COMMISSION 12: RADIATION AND STRUCTURE OF THE SOLAR ATMOSPHERE \\ (RADIATION ET STRUCTURE DE L'ATMOSPHERE SOLAIRE)
}

\author{
Report of Meetings, 22, 24 and 25 August 1970
}

Chairman and Acting President: R. Grant Athay.

SECRETARY: L. Delbouille.

\section{Business Session}

The chairman gave first a welcoming address and best wishes for the work of the Commission in the name of Dr Gnevyshev, who was unable to attend the General Assembly.

He then transmitted an inquiry from the General Secretary of the IAU asking that the commission inform the General Secretary of the manner in which the commission arrives at official decisions.

The new officers of the Commission were then proposed to the members, and approved by a vote.

The chairman then read a proposed set of rules for the operation of Commission 12. After some discussion it was decided that a formal set of rules should not be adopted until those members of Commission 12 not in attendance at Brighton had had an opportunity to study them. The chairman agreed to circulate the proposed rules to all members of the commission for their critical comment.

The chairman then asked the members if they had questions to raise.

de Jager: Is it worthwhile to plan a new 'Bilderberg Conference?'

\section{Answers:}

Bonnet: Yes, it gives good opportunities for contacts between experimenters and theoreticians. Giovanelli: Yes, but some thought should be given as to how far in the chromosphere to push the model.

Namba: Is it not time now to include some non-homogeneous structure?

Wilson: Perhaps we should have a special meeting on inhomogeneities.

Jefferies: The first Bilderberg conference was very stimulating. I am personally very favorable to another meeting in 2 or $3 \mathrm{yr}$.

Athay: It is important to have, at all times, a reference model to which a large number of people have contributed. One of the most useful features of the Bilderberg model was that enough people contributed to the model that it became a genuine reference model.

Athay: Have you any suggestions about colloquia or symposia to be organized by Commission 12? Plans are already in progress for a joint discussion, in Sydney, on solar fine structure. This will involve several commissions besides Commission 12.

Athay: Another question we need to discuss is that relative to the reports, both the past one as published and the next one, to be prepared for 1973. The general guidelines for the past report were that it should be very short, should not include references to work already published, and should not include a bibliography. It seems that a bit more freedom will be given for the next one. Three points were then discussed:

(a) Bibliography in the future report.

The strong consensus was that all future reports should contain complete bibliographies.

Houtgast introduced an idea given by Thomas at Commission 36 .

Thomas gave more particulars suggesting a distribution, through the president, at the end of each year of a neswletter including the titles only (or the titles plus abstracts) of all the papers brought to the attention of the commission president. The newsletter can probably be distributed even to interested people not in the Commission. 
Newkirk asked about the possibility to distribute, as in the past, the report first in a draft form. This was not possible for the present report.

(b) In the past, the report was supposed to mention the work made between two IAU Assemblies. What is the opinion of the members on this question?

Pasachoff favors the inclusion of all the work done during each three year period.

Newkirk - Inclusion of only the work in progress attenuates the usefulness of the report, which was, in the past, an easy source of references.

Other members of the Commission also strongly favored this point of view.

(c) Should the report include evaluated comments by its author?

Thomas: Giving a personal opinion is always dangerous. In any case, nothing should be suppressed by a personal choice.

Orrall insists once more on the usefulness, if practical, to include a complete bibliography.

Thomas: Perhaps in the form of a separate report?

Athay: A separate report can more easily be lost. What about the use of 'Astronomy Abstracts,' giving only their number and title?

Müller agrees with Thomas about the difficulty to be objective, and asks if the report should not be just a listing of work done, without any comment.

Newkirk agrees also. It seems to him that the IAU reports are not the most appropriate place for comments.

Houtgast stresses the point that excluding comments will shorten the report, and take less pages in the printed book.

Athay concludes the discussion in accepting the idea that comments should be reduced to a minimum.

\section{Other problems raised:}

Orrall: What can be done about common interests of Commission 12 with Commission 10, perhaps also with Commissions 40,44 and 36 ?

Athay announces his intention to propose that Commissions 10 and 12 work jointly in organizing their scientific sessions. This will be proposed and discussed in a joint meeting of the Organizing Committees of the two Commissions a few days later.

Pierce suggested that the Commission should discontinue the work of the group on central line intensities, but should create two new Working Groups:

(a) On sunspots spectra (excluding magnetic fields problems).

(b) On the high resolution atlas of the photospheric spectrum.

He suggests Delbouille as chairman for this second new group.

Jefferies expressed his interest in such a group, insisting on the usefulness for its members to include theoreticians, as advisers about what has to be done.

He suggests Pierce as chairman of a new working group on sunspots spectra, and Pierce accepts, on the condition that the 'central intensities' group be discontinued.

A vote of approval was cast for the formation of the two new groups:

(a) On sunspots spectra (Chairman A. K. Pierce),

(b) On a high resolution atlas of the photospheric spectrum (Chairman L. Delbouille) and for the dissolution of the previous group on central intensities.

\section{SCIENTIFIC COMMUNICATIONS}

A. Wyller: 'Fabry-Pérot Observations of Sunspots'.

A scanning type Fabry-Pérot filter with a band pass of $0.04 \AA$ in the $\mathrm{Na} D$ line region has been built for observations of sunspots.

C. E. Moore-Sitterly: 'The Revision of the Multiplet Tables'.

This was mainly an announcement that the Section III (CI to CVI) is now in press, and the Section IV $(\mathrm{N})$ is in preparation. 
P. Lemaire (presented by R. M. Bonnet): 'High Resolution Spectra of the Mgn Resonance Lines Observed from a Balloon-Borne Instrument.'

To observe the MgI lines, a balloon-borne instrument was built, comprising a cassegrain telescope $(F / 20$, focal length $300 \mathrm{~cm}$ ) and a spectrograph with a theoretical resolution of about $10 \mathrm{~m} \AA$. In flight, $25 \mathrm{~m} \AA$ spectral and $3 \mathrm{~s}$ spatial resolutions have been achieved. The spectra show strong local variations on the solar disk. In general the shape of the lines is asymmetric and seems single peaked across the spots.

\section{Second Meeting}

Chairman: E. A. Müller.

A. Dupree and L. Goldberg: 'New Information on the Structure of the Quiet Chromosphere and Corona from OSO IV and OSO VI Data.'

A report was given based on the two OSO satellites (observations between 300 and $1400 \AA$ with about $3 \AA$ resolution) and on a 1969 rocket experiment with a photoelectric scanning spectrometer for the region 1350 to $1825 \AA$. A model of the chromosphere, the transition region and the corona has been built to fit these observations.

R. J. Bray: 'Preliminary Observations of the Chromosphere with a Computer-Controlled Tunable $1 / 8 \AA$ Filter.'

This filter is installed in the $30 \mathrm{~cm}$ chromospheric telescope of the CSIRO Solar Observatory (Australia).

V. N. Karpinsky: 'Some Results of Solar Granulation Photography in Pulkovo Observatory.'

A resolution of 0.3 to $0.5 \mathrm{arc} \mathrm{sec}$ has been achieved in various monochromatic pictures of the solar granulation. Very good correlation is found between images taken simultaneously at 4800 and $6000 \AA$.

V. A. Krat: 'Granulation Studies with Balloon-Borne Instruments in Pulkovo Observatory.'

Two successful flights took place, one at the end of 1966 and one in 1970. For the 1970 flight, observations lasted six hours from an altitude of $20.5 \mathrm{~km}$. About $6 \%$ of the 900 pictures obtained show a resolution corresponding to the theoretical diffraction limit of of the $50 \mathrm{~cm}$ objective. An attempt to measure the $\mathrm{D} \alpha$ deuterium line in the blue wing of $\mathrm{H} \alpha$ in the absence of most of the telluric $\mathrm{H}_{2} \mathrm{O}$ absorption leads to a value of $\mathrm{D} / \mathrm{H}<2 \times 10^{-5}$.

J. Rösch: 'Images recentes de taches solaires.'

Various exposure times give the possibility to see many detailed features at different brightness levels between the chromosphere and the central region of the spots.

P. R. Wilson and C. J. Cannon: 'Velocity and Brightness Fluctuation Correlations in Chromospheric Lines.'

Report of line-center intensities and velocity fluctuations measured for $\mathrm{Mg} \mathrm{b}, \mathrm{Na} \mathrm{D}$ and $\mathrm{Can} \mathrm{K}$ lines. Near the limb, the strucuture is consistent with the supergranulation cells. At disk center, regions of significant correlation may extend over distances of the order of $25000 \mathrm{~km}$.

There are points on the disk at which the $\mathrm{K}_{2}$ emission feature appears only in either the red or the blue wing of the line, but also regions in which the doubly peaked profile is real.

Z. Suemoto: 'Emission Line Profiles and the Inhomogeneous Model of the Chromosphere.'

High spatial resolution spectrograms of the Ca II $\mathrm{K}$ line suggests that the averaged line profile may result from a more or less random distribution of many narrow local emission and absorption features.

\section{Third Meeting}

Chairman: Z. Suemoto.

V. N. Karpinsky: 'The High Precision Investigation of Fraunhofer Lines in Pulkovo Observatory.'

The Pulkovo Observatory is equipped with a photoelectric high resolution solar spectrometer used since 1966 to record line profiles and central intensities in the visible.

L. Delbouille, G. Roland, and R. Zander: 'Recent High Resolution Solar Spectroscopy by the Liège Group." 
Tables of wavelengths and identifications of the 10450 lines measured in the Atlas of the Solar Spectrum between $\lambda 7498$ to $\lambda 12016$ published in 1963 have been prepared by W. S. Benedict and J. W. Swensson and are under print. With the collaboration of L. Neven, the Jungfraujoch photoelectric spectrometer is currently used to remap the photospheric spectrum between 3000 and $12000 \AA$. The region 5000 to $6300 \AA$ is ready to be printed. A balloon-borne infrared spectrometer was flown in April 1970. The solar spectrum between 1.8255 and $1.8570 \mu$ has been recorded with a resolution limit better than $0.04 \mathrm{~cm}^{-1} .72$ water lines and 67 solar lines have been measured in this domain.

N. Grevesse: 'New Solar Abundances and Identifications Obtained at Liège.'

The following results were recently obtained:

(a) Solar abundances of Ni from the photospheric [NirI] lines by N. Grevesse and J. P. Swings $\left(\log N_{\mathrm{N} 1}=6.30\right)-$ Solar Phys. 13, 19, 1970.

(b) [FeI] lines: their transition probabilities and occurrence in sunspots, by N. Grevesse, H. Nussbaumer and J. P. Swings $\left(\log N_{\mathrm{Fc}}=7.50\right)$.

(c) Identification of $\mathrm{SiH}^{+}$in the solar photospheric spectrum, by N. Grevesse and A. J. Sauval.

(d) Oscillator strengths for $\mathrm{SiH}$ and $\mathrm{SiH}^{+}$deduced from the solar spectrum, by $\mathrm{N}$. Grevesse and A. J. Sauval.

(e) Solar and meteoritic abundances of mercury, by N. Grevesse.

(f) Deuterium in the solar photospheric spectrum, by N. Grevesse. To appear in the proceedings of the 16th Liège Colloquium.

E. A. Müller: 'On the Solar Lithium and Beryllium Abundances.'

E. A. Müller reported on a detailed investigation of the solar lithium feature carried out in collaboration with J. W. Brault and E. Peytremann. They obtain $\log \varepsilon_{\mathrm{Li}}=1.0 \pm 0.10$ and $\mathrm{Li}^{7} / \mathrm{Li}^{6} \geqslant 10$. Center-limb observations secured by Brault and Müller of the solar absorption features at $\lambda 3321$ and $\lambda 3130-3131$, and of the lines of BeI and Ben recorded with a laboratory source were presented. The solar feature at $\lambda 3321$ proved not due to BeI. Both BeII resonance lines are present, but severely blended, in the solar spectrum. Both the equivalent width and the abundance of beryllium in the Sun have up to now been much overestimated. This work is still in progress.

G. Brückner: 'Line Profiles in the Extreme UV.'

Recent results obtained at the Naval Research Laboratory with high resolution far UV spectrometers were reported.

H. L. Demastus and R. B. Dunn: 'Coronal Movies.'

Two movies were shown containing the best coronal scenes taken from the Sacramento Peak Observatory between October 1966 and November 1969.

The scenes contained a number of flare-associated fast-accelerated expansions and also several fine loops.

\section{Fourth Meeting}

Chairman: A. K. Pierce.

D. Hall: 'Observations of the Infrared Sunspot Spectrum between 1 and $2.5 \mu$.'

The infrared umbral spectrum has been observed, at Kitt Peak National Observatory, in the atmospheric windows $\lambda 11340-\lambda 13528, \lambda 14872-\lambda 18037$ and $\lambda 19614-\lambda 24778$, with a spectral resolution of 100000 .

Preliminary analysis of the spectra has established the presence of umbral lines due to the molecules $\mathrm{OH}, \mathrm{CO}, \mathrm{CN}, \mathrm{HF}$ and $\mathrm{H}_{2} \mathrm{O}$.

J. W. Harvey: 'A Photographic Sunspot Spectrum Atlas ( $\lambda$ 3700- $\lambda$ 9200).'

A photographic atlas of the spectrum of a large sunspot is in preparation at Kitt Peak National Observatory.

G. Gonczi and F. Roddier: 'A Model of the Solar Photospheric Velocity Field.'

The model presented includes two layers of different microturbulence and, for convection, an ascending and a descending columns.

H. Wöhl: 'Photoelectrically Scanned Sunspot Spectra $\lambda 4000$ to $\lambda 8000$.' 
Newly identified molecules in the sunspot spectra are $\mathrm{NiH}$ and $\mathrm{CoH}$. Another similar sunspot spectrum was obtained by H. Wöhl, A. Wittman and E. H. Schröter (Gottingen Univ. Observatory). A section of the uncorrected spectrum $(\lambda 4500-\lambda 7000)$ is available on magnetic tape.

O. Hauge: 'The Presence of CuH Lines in the Sunspot Spectrum and the Solar Isotope Ratio of Copper.'

$\mathrm{CuH}$ lines from the $\mathrm{R}$ and $\mathrm{P}$ branches of the ${ }^{1} \Sigma-X^{1} \Sigma(0,0)$ band are shown to exist in the sunspot spectra obtained at the Kitt Peak National Observatory. From two CuI lines of relatively high isotope splitting, an isotope ratio $\mathrm{Cu}^{63} / \mathrm{Cu}^{65}=2 \pm 1$ is obtained.

P. Turon: 'High Resolution Sunspot Images at $10 \mu$.'

A slow raster scanning technique with a germanium bolometer has been used to build TV images of the Sun at $10 \mu$. The Kitt Peak National Observatory solar telescope allows a diffraction limited resolution of 2 arc sec.

O. Namba and G. Hafkenscheid: 'Profiles of the CI 5052 Line from Individual Elements of the Granulation.'

This line is appreciably stronger in the granules than in the intergranular regions, but the outer parts of the profiles are broader in the intergranular regions than in the granules. The profiles from the intergranular regions are red-shifted relative to those from the granules by 0 to $0.6 \mathrm{~km} / \mathrm{sec}$.

\section{WORKING GROUP ON SOLAR ECLIPSES}

Chairman: M. Rigutti.

D. H. Menzel and J. M. Pasachoff reported on prospective sites for the 30 June 1973 solar eclipse in Africa. The sites fall in the Sahara desert and the Air mountains in Niger and Mali. They suggest, because of the limited facilities available there, that substantial IAU-coordination on an international basis would be advisable, and that local preparations must be commenced long in advance. Preliminary weather forecasts are most favorable in the visited sites.

\section{ECLIPSE OF MARCH 7, 1970}

J. Houtgast reported on observations made by the Dutch party at Miahuatlan, Mexico. A Littrow type slit spectrograph with a resolution of 150000 equipped with slit jaw camera for the orientation of the solar image, a photoelectric exposure time device, and a photoelectric multiplexing device in the $\mathrm{Mg} \mathrm{b}$ region were used.

B. Fort reported on an experiment with a specially built new Lallemand type electronic camera made by the 'electronic camera group' of the Meudon Observatory. The observations were made by direct photography through a very narrow band pass filter. Brightness distribution of both intensity and polarization of the green line up to a height of about 1.25 solar radii with a good spatial resolution was obtained.

Z. Suemoto reported on observations made by the Japanese party at Puerto Escondido, Mexico. A quadruple camera (focal length of $5 \mathrm{~m}$ ) with a Newkirk filter and four polaroids just in front of the film was used by $\mathrm{K}$. Saito in order to study three-dimensional structures even in the innermost corona. M. Makita took direct photographs (focal length of $5 \mathrm{~m}$ ) with a continuous spectrum of about $100 \AA$ centered around $6900 \AA$ excluding the $\mathrm{H} \alpha$-lines of hydrogen and the $6678 \AA$-line of HeI. M. Kanno got a number of flash spectra in the range from 4686 to $6678 \AA$ with an oblique incidence slitless grating spectrograph and a solar image of $46 \mathrm{~mm}$ in diameter.

T. C. Van Flandern reported on observations from near the path edge made by himself, D. E. Dunham and J. E. Bixby.

M. Waldmeier presented a documentary film on the instrumentation of a number of observing parties and on the partial and totality phase of the eclipse.

V. Gaizauskas provided the audience with copies of a map prepared by the Canadian N.R.C. 
Astrophysics Branch of the totality path through Canada of the total eclipse of $10 \mathrm{July} 1972$. He announced also the preparation of tables for weather forecasts.

Z. Suemoto announced the preparation by the National Meteorological Bureau of Japan of weather forecasts for the 30 June 1973 eclipse in Africa.

The Chairman gave a short report on the activity of the Working Group on Solar Eclipses.

First of all he remarked on the impossibility of preparing a report on the past 1968 and 1970 eclipses with a summary of the main subjects covered by successful experiments because of the very limited information provided to him by the party leaders. For this reason the last issue of the beautiful 1970 eclipse bulletin, edited by the U.S. Coordinator Dr E. Belon, will be the only comprehensive source of information of successful observations during the 1970 eclipse.

After this, the Chairman remarked on the rather poor activity of the Working Group in the past three years and asked the members and the audience to reconsider the usefulness of the existence of the Working Group itself. A long discussion took place and the following points were unamimously reached.

1. The Working group on Solar Eclipses must continue its work which can be very useful to all solar astronomers. Accordingly, the members will actively participate in the needs of the group. New members willing to join the group should be accepted and old ones not really active should be cancelled.

2. The Group should get a representation in the COSPAR.

As far as the activity of the working group in the near future is concerned, it has considered the possibility of organizing a symposium on 'Theoretical and experimental problems related to solar eclipse observations.' This symposium should be an occasion to discuss problems both from the theoretical and experimental point of view and would have the aim of pointing out the most interesting problems which can be considered for experiments during solar eclipses, and to suggest to solar astronomers what kind of observations could be worthwhile to acquire. It came out from the discussion that such a symposium should interest a great number of people and that, for this reason, it should be necessary to find a supporting organization and a host country. G. Athay, President of Commission 12, will then contact the COSPAR and the IUCSTP for trying to get the necessary support.

The usefulness of preparing a comprehensive bibliography of as many as possible papers appearing as a consequence of solar eclipse experiments and studies has been examined. The Chairman will test the actual feasibility of such a bibliography by inquiring personally and through the IAU Bulletin among solar astronomers.

The need for information about the next eclipses has been recognized. Contacts with governments of countries through which the total eclipse path will pass will be necessary but it appeared clear that it will be wise to establish them through important organizations. G. Athay will ask the U.S. National Science Foundation whether they will continue to act as an information centre. This problem is, of course, of the greatest importance for the 1973 eclipse.

Concluding the meeting, the Chairman on behalf of the whole group expressed thanks to Dr E. Belon and the people who worked with him for the publication of the 1970 eclipse bulletin, $\mathrm{Dr}$ E. R. Dyer Jr., secretary of the IUCSTP, Dr G. Haro, Dr J. L. Locke, Dr A. de Tuddo, co-director of the 'Estudio de los recursos del Estado de Oaxaca' and all the persons who in one way or another made easier for the observers the work connected with the eclipse experiments in 1968 and 1970.

\section{WORKING GROUP ON SUNSPOT SPECTRA}

Chairman: A. K. Pierce.

An organizational meeting of the working group on the spectra of sunspots was held at Brighton, England. All interested persons are invited to participate in the activities of the group. The work of the group will primarily be in the exchange of information with respect to: atlases (photographic, photoelectric), identifications, wavelengths, models, and abundances. 


\section{WORKING GROUP ON CENTRAL LINE INTENSITIES}

Chairman: A. K. Pierce.

The preliminary results of a number of workers have been brought together. The following tabulation is a partial listing of corrected central intensities together with their continuum points. Further details are to be found in the literature or from: Goldberg-Rogozinskaya, Karpinsky, Krat, Pravdjuk, Babiy, Gurtovenko, White, Delbouille, Brault, or Pierce.

\begin{tabular}{|c|c|c|c|c|c|}
\hline \multirow[t]{3}{*}{$\mathrm{Ni}$} & 3411.500 & 100.0 & $\mathrm{Fe}$ & 5574.796 & 100.0 \\
\hline & 3414.799 & 2.3 & & 5576.099 & 22.2 \\
\hline & 3419.932 & 100.0 & & 5577.960 & 100.0 \\
\hline \multirow{3}{*}{$Y_{\text {II }}$} & 3600.525 & 98.4 & $\mathrm{Ni}$ & 5846.882 & 99.7 \\
\hline & 3600.739 & 9.4 & & 5847.006 & 76.9 \\
\hline & 3601.113 & 99.8 & & 5849.395 & 100.0 \\
\hline \multirow[t]{3}{*}{$\mathrm{Fe}$} & 4069.424 & 100.0 & $\mathrm{Na}$ & 5888.220 & 96.4 \\
\hline & 4071.749 & 3.2 & & 5889.973 & 4.1 \\
\hline & 4073.401 & 98.1 & & 5894.000 & 98.0 \\
\hline \multirow[t]{3}{*}{$\mathrm{Ca}$} & 4220.909 & 100.0 & $\mathrm{Na}$ & 5894.000 & 98.0 \\
\hline & 4226.740 & 2.3 & & 5895.940 & 4.8 \\
\hline & 4230.803 & 100.0 & & 5898.620 & 98.6 \\
\hline \multirow[t]{2}{*}{ Mg } & 4571.104 & 14.5 & $\mathrm{Fe}$ & 6085.818 & 99.9 \\
\hline & & & & 6089.574 & 64.1 \\
\hline $\mathbf{M g}$ & 5167.330 & 8.0 & & 6089.881 & 99.9 \\
\hline \multirow[t]{2}{*}{$\mathrm{Mg}$} & 5172.700 & 5.8 & $\mathbf{N i}$ & 6128.751 & 100.0 \\
\hline & & & & 6128.984 & 72.1 \\
\hline $\mathbf{M g}$ & 5183.621 & 5.2 & & 6130.482 & 100.0 \\
\hline \multirow[t]{3}{*}{$\mathrm{Fe}$} & 5294.932 & 99.9 & $\mathrm{Ni}$ & 6128.751 & 100.0 \\
\hline & 5295.321 & 66.7 & & 6130.141 & 77.6 \\
\hline & 5295.556 & 99.8 & & 6130.482 & 100.0 \\
\hline \multirow[t]{3}{*}{$\mathrm{Fe}$} & 5304.708 & 100.0 & $\mathrm{Fe}$ & 6677.357 & 100.0 \\
\hline & 5307.369 & 21.7 & & 6677.997 & 27.5 \\
\hline & 5308.163 & 100.0 & & 6679.088 & 99.9 \\
\hline \multirow{4}{*}{$\mathrm{Ni}$} & 5432.170 & 100.0 & $\mathrm{Ni}$ & 6767.120 & 100.0 \\
\hline & 5435.866 & 46.0 & & 6767.784 & 32.9 \\
\hline & 5440.334 & 100.0 & & 6768.205 & 100.0 \\
\hline & & & $\mathrm{O}_{2}$ & $\begin{array}{l}6870-6877 \text { zero } \\
\text { air masses. }\end{array}$ & tensity at 5 \\
\hline
\end{tabular}

\section{WORKING GROUP ON THE HIGH RESOLUTION ATLAS OF THE PHOTOSPHERIC SPECTRUM}

Chairman: L. Delbouille.

A first informal meeting to organize the working group was held in Brighton. A first list of persons interested in its activity has been made, but can, of course, always be expanded in the future. After discussion, a few guidelines have been established for the activities of the group, whose main purpose is to coordinate observational work made by several groups and to try to organize the centralization of all the assignment work in a single location. As a first scheme, it has been recognized that the Liège group, from the Jungfraujoch high altitude station, is progressing in the record of the region $\lambda 3000-\lambda 12000 \AA$ and will publish it in sections, with wavelength calibration done in collaboration with Kitt Peak National Observatory. Coordination of future work further in the infrared will be attempted as soon as possible. Contacts will be taken by Dr Delbouille about the possibility to concentrate all the work on the assignments of all the measured lines in Washington, D.C., U.S.A. Practical exchange of information will be made in the form of newsletters by the chairman and private communications to him. 